\title{
Constructing anisotropic finite element model of bone from computed tomography (CT)
}

\author{
Siamak Kazembakhshi and Yunhua Luo* \\ Department of Mechanical Engineering, Faculty of Engineering, University of Manitoba, Winnipeg, \\ MB R3T 5V6, Canada
}

\begin{abstract}
Image-based finite element (FE) modeling of human bones has been increasingly applied as a useful tool in biomedical engineering. However, most existing image-based FE models assume isotropic mechanical properties for bones, although bones are typically anisotropic material. In this study, we attempted to construct anisotropic FE models from medical computed tomography (CT) scans by modifying the existing empirical relations of bone elasticity-density. The hypothesis adopted in the study is that bone anisotropy is generated by the variations of bone density and the proposed anisotropic relations should degenerate to the isotropic ones if bone density variation is taken zero. The effect of considering bone anisotropy in FE models was investigated by numerical studies. The obtained numerical results showed that the relative error in the finite element solutions produced respectively by the isotropic and anisotropic FE models can be as large as $50 \%$. We concluded from this preliminary study that the consideration of anisotropy in bone FE models has a significant effect on the accuracy of bone behavior predicted by the FE models. However, well-designed bone tests have to be conducted to validate the anisotropic bone elasticity-density relation proposed in this study.
\end{abstract}

Keywords: bone, finite element model, computed tomography, anisotropy, elasticity modulus

\section{Introduction}

Image-based finite element modeling of human bones has been increasingly applied in biomechanical and biomedical engineering. Two examples of the applications are injury analysis and pre-surgery simulation. Human bones are typical anisotropic and heterogeneous material [1-12], characterized by large variations in their physical and mechanical properties [1,6-8]. Bone properties are dependent on the age, gender, health status, and skeletal site of the subject [1,11-13]. Therefore, subject-specific finite element models must be constructed in the applications. Computed Tomography (CT) is a threedimensional (3D) medical imaging modality for capturing tissue information in in-vivo human body. The information required for constructing a finite element model can be extracted from CT images. The geometric information in the CT images is used in generating a finite element mesh. The variations in the CT numbers or Hounsfield Units (HU) are related to tissue physical and mechanical prop-

\footnotetext{
${ }^{*}$ Corresponding author: Yunhua Luo, Department of Mechanical Engineering, Faculty of Engineering, University of Manitoba, Winnipeg, MB R3T 5V6, Canada. Tel.: 1 (204) 474 6899; Fax: 1 (204) 275 7507; E-mail: Yunhua.Luo@umanitoba.ca.
}

0959-2989/14/\$27.50 @ 2014 - IOS Press and the authors. 
erties by empirical functions [14-17]. These functions have been established by a large amount of in vitro experiments. For example, bone density is related to CT number by linear functions $[12,14]$. Bone elasticity, or Young's modulus, is related to bone density by exponential or linear functions [13]. The above relations are a necessary component in constructing finite element models. However, the existing empirical functions are only useful in constructing isotropic finite element models. In most existing bone elasticity-density functions, bone density is the only variable required to determine the elasticity. The orientation dependence of bone elasticity, i.e., bone anisotropy, has not been considered. In this study, we attempted to modify existing bone elasticity-density empirical functions to consider bone anisotropy. The challenge is in relating the bone anisotropy to CT number or Hounsfield Unit (HU) in CT scans. On the basis of the research done by Schneider et al. [15], we made the following hypothesis: bone anisotropy is generated by the variations of bone density and the obtained anisotropic elasticity-density relations should degenerate to the isotropic ones if bone density variation is taken zero. Bone specimens were virtually taken from different locations of the femur bone using clinical CT images. Paired isotropic and anisotropic finite element models were constructed for these specimens. The effect of adopting isotropic or anisotropic material model was investigated by applying the same boundary and loading conditions to paired models, and studying the differences in the predicted stresses/strains.

\section{Materials and methods}

The gradient of bone density is used to define the principal orientation of the bone anisotropy. Bone density is linearly related to Hounsfield Unit (HU) by,

$$
\rho\left(\frac{g}{c m^{3}}\right)=a \times H U+b
$$

Different coefficients $a$ and $b$ have been reported in the literature [16,17]. The HU value is converted from CT number by assigning $-1000 \mathrm{HU}$ to air and $0 \mathrm{HU}$ to water. The HU value for segmenting bone from soft tissue is taken as $-115 \mathrm{HU}$ as proposed in [15].

\subsection{Variation of bone density within voxel}

In a CT voxel, the CT number is a constant and so is the bone density. However, if a higher resolution is used, i.e., the CT voxel is split into small ones, the smaller voxels may have different bone density. In this study, bone density is considered as a continuous function within a voxel. The function is constructed using an interpolation method, for example, the local multivariate Lagrange interpolation method [18], and density data from neighboring voxels.

Consider a typical voxel in CT image (see Figure 1), the bone density within the voxel can be expressed as

$$
\rho(x, y, z)=\rho_{n}+\rho_{x}^{\prime} \cdot x+\rho_{y}^{\prime} \cdot y+\rho_{z}^{\prime} \cdot z
$$




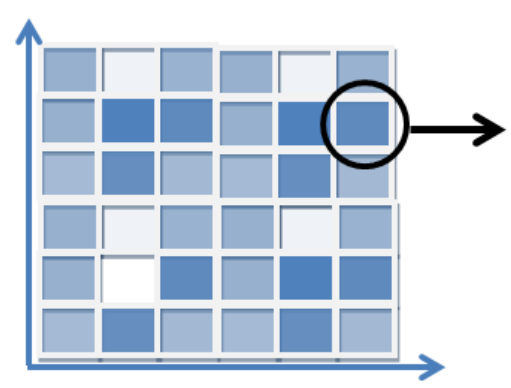

(a)

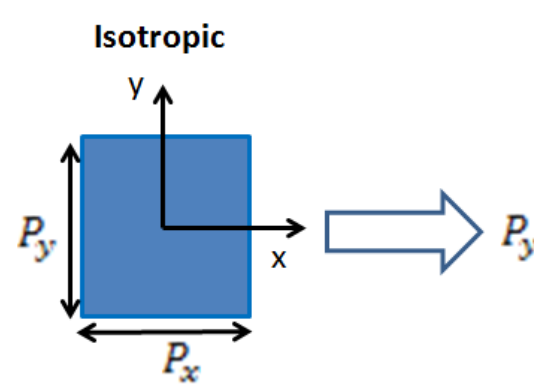

(b)

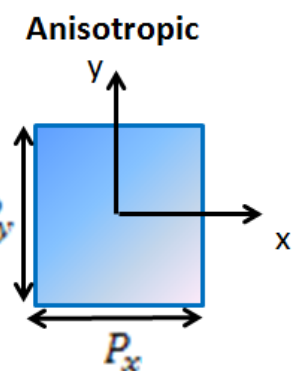

(c)

Fig. 1. (a) Zoomed CT image; (b) voxel with constant density; and (c) voxel with variable density.

where $\rho_{x}^{\prime}, \rho_{y}^{\prime}$, and $\rho_{z}^{\prime}$ are the three components of the bone density gradient vector; $x, y$ and $z$ are the coordinates of a concerned point within the voxel, and $\frac{-P_{x}}{2} \leq x \leq \frac{P_{x}}{2}, \frac{-P_{y}}{2} \leq y \leq \frac{P_{y}}{2}, \frac{-P_{z}}{2} \leq z \leq \frac{P_{z}}{2}$; $P_{x}, P_{y}$ and $P_{z}$ are the voxel dimensions.

\subsection{Principal anisotropy orientations}

Bone anisotropy is generated by its density variation and the maximum variation of bone density represents the maximum anisotropy [15]. Therefore, the first-principal orientation at a point is given by the gradient vector of bone density (see Figure 2), $\hat{e}_{1}=\nabla \rho=\left[\rho_{x}^{\prime} \rho_{y}^{\prime} \rho_{z}^{\prime}\right]$, always pointing to the increasing direction. Then, a plane $(S)$ perpendicular to $\hat{e}_{1}$ is defined. The second axis $\left(\hat{e}_{2}\right)$ is obtained by projecting the global $X$-axis onto the plane $(S)$. If $X$-axis is normal to the plane, then use the projection of $Y$-axis. The third axis $\left(\hat{e}_{3}\right)$ is determined using the right-hand rule. If the global base vectors are denoted $\hat{\imath}, \hat{\jmath}$, and $\hat{k}$, then a transformation matrix $(T)$ between the two sets of coordinates can be defined as

$$
T=\left[\hat{e}_{1} \hat{e}_{2} \hat{e}_{3}\right]^{T} \cdot\left[\begin{array}{lll}
\hat{\imath} & \hat{\jmath} & \hat{k}
\end{array}\right]
$$

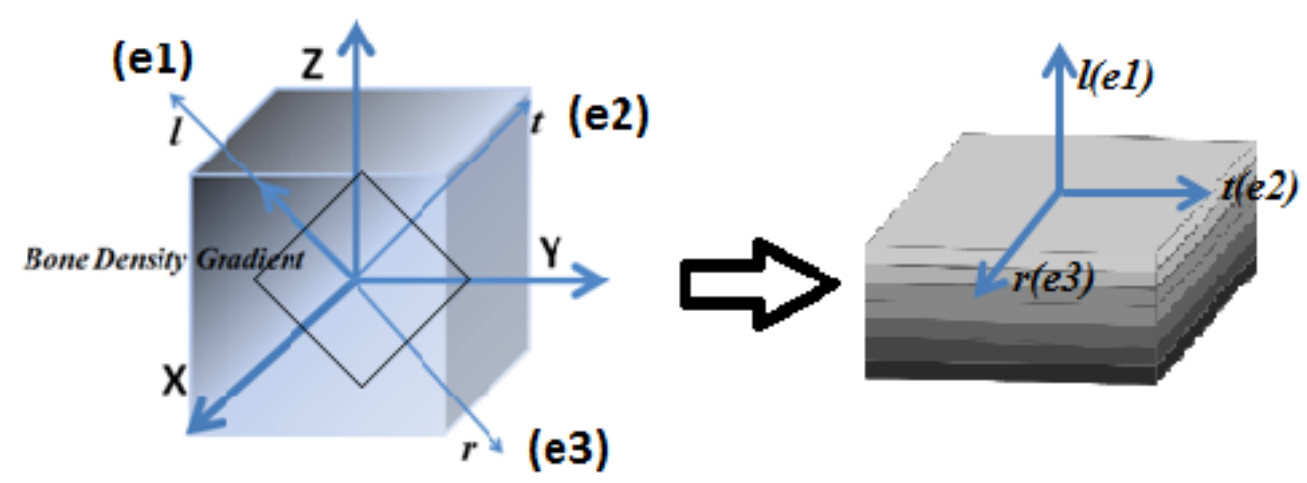

Fig. 2. Global and principal anisotropy. 


\subsection{Anisotropic elasticity constants}

The general constitutive equation for bone is

$$
\sigma=D \varepsilon
$$

where $\sigma$ is the stress tensor, $D$ is elasticity tensor and $\varepsilon$ is the strain tensor. By following Schneider [15], bone is considered as an orthotropic material, then

$$
\begin{aligned}
& E_{1}=E_{a}, E_{2}=E_{3}=E_{p} \\
& v_{12}=v_{13}=v_{a p}, v_{21}=v_{31}=v_{p a}, v_{23}=v_{p} \\
& G_{12}=G_{13}=G_{a}, G_{23}=G_{p}
\end{aligned}
$$

where $a$ and $p$ stand for axis of symmetry direction and in-plane of isotropy, respectively. $v_{a p}$ and $v_{p}$ are related by,

$$
\frac{v_{a p}}{E_{a}}=\frac{v_{p}}{E_{p}}
$$

Poisson's ratios $v_{a p}$ and $v_{p}$ are considered as independent of bone density and their values can be directly taken from experimental data [1]. The shear moduli are expressed as

$$
G_{a}=\frac{E_{a}}{2\left(1+v_{a p}\right)}, \quad G_{p}=\frac{E_{p}}{2\left(1+v_{p}\right)}
$$

By observing the expressions in Eqs. (5)-(7), the key to extending the existing bone elasticitydensity relations to consider the bone anisotropy is to relate the two elasticity moduli, $E_{a}$ and $E_{p}$, to bone density and its gradient. Based on the assumptions made before, the only change that should be made is in $E_{a}$; In the isotropic plane, the bone density has no variation, therefore, $E_{p}$ should not be changed. There are many bone elasticity-density relations in the literature [19]. If the following generic elasticity-density expression is taken,

$$
E=f(\rho)
$$

then, the anisotropic expressions are

$$
E_{a}=f\left(\rho_{0}+|\nabla \rho| \cdot \bar{P} / 2\right), \quad E_{p}=f\left(\rho_{0}\right)
$$




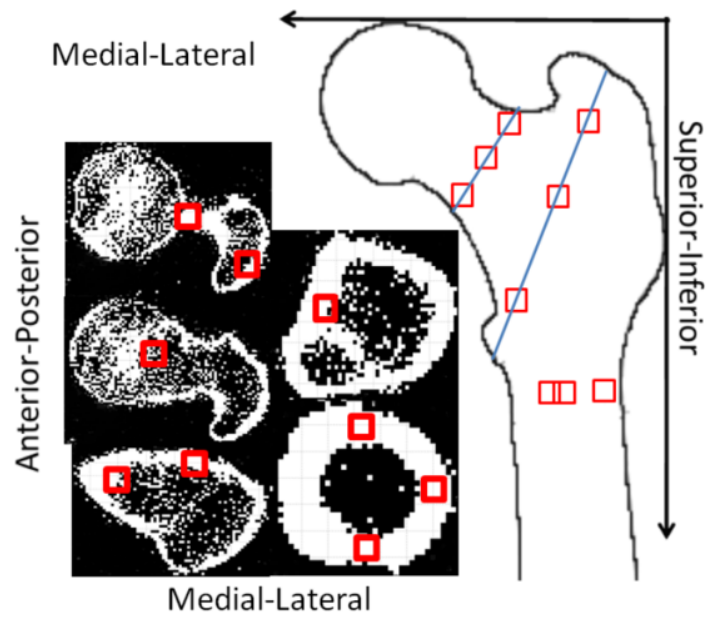

Fig. 3. Locations of virtual specimens.

where $\rho_{0}$ is the bone density at the voxel center; $\bar{P}=\left(P_{x}+P_{y}+P_{z}\right) / 3$ is the average dimension of a voxel in CT image. If the gradient of bone density is zero, the expressions in Eq. (9) will degenerate back to the one in Eq. (8).

\section{Numerical investigations}

The effect of the adopted bone material model, isotropic or anisotropic, on the predicted stress/strain level in the bone was investigated. Nine specimens were virtually extracted from clinical CT images of a human femur (see Figure 3), at the femoral neck, the intertrochanter and the shaft; the specimens symmetrically stride across the sagittal middle-plane. The surfaces of the specimens are taken parallel to the global coordinate planes. The specimens have cubic shape and dimensions of $5 \mathrm{~mm}$.

In the numerical investigations, the relation of bone density and Hounsfield units proposed in $[16,17]$ was considered,

$$
\rho\left(\frac{g}{c m^{3}}\right)=0.0012 \times H U+0.17
$$

The Poisson's ratio was assumed to be independent of bone density [15]. The isotropic bone elasticity-density correlations were taken from the literature [16,17],

$$
\begin{array}{ll}
\rho<1.04\left(\frac{\mathrm{g}}{\mathrm{cm}^{3}}\right) & E_{T}(M P a)=1310 \rho^{1.4} \\
\rho>1.04\left(\frac{\mathrm{g}}{\mathrm{cm}^{3}}\right) & E_{C}(M P a)=14261 \rho-13430
\end{array}
$$

where the subscripts $T$ and $C$ represent trabecular bone and cortical bone, respectively. The above expressions were modified based on Eqs. (5)-(7) and (9) to obtain the corresponding relations for the 
anisotropic model. Finite element modeling was conducted using commercial software Abaqus. Eightnode cubic solid element was used in the simulations. The inhomogeneous anisotropic material properties were assigned at element nodes using the temperature and field variable (FV) as an auxiliary tool. For the paired isotropic and anisotropic finite element models, the same finite element mesh, constraint and loading (axial direction) conditions were used in the simulations. The only difference between them is in the material model. The difference was measured by

$$
d(\%)=\frac{\left|f_{\text {aniso }}-f_{\text {iso }}\right|}{\left(\left|f_{\text {aniso }}+f_{\text {iso }}\right|\right) / 2}
$$

Where $f_{\text {aniso }}$ and $f_{\text {iso }}$ are, respectively, stress (or strain) predicted by the anisotropic and isotropic finite element models.

\section{Results and discussions}

The distribution of the first-principal elasticity modulus for the anisotropic finite element models is displayed in Figure 4. From the figure, it can be seen that in general the cortical bone has larger elasticity modulus than the trabecular bone. For the cortical bone at the femur shaft, the orientation of the first-principal elasticity modulus is roughly parallel to the shaft axis, which is consistent with experiment observations [1]. However, the orientation is different from point to point, suggesting that bone anisotropy is best defined and described pointwise.

The differences in the stresses (and strains) predicted by the finite element models with isotropic and anisotropic material model are presented in Table 1. The compared variables include the maximum von Mises stress and the maximum first principal strain, as they are usually used in material failure criteria. A visual comparison between the two types of finite element models is displayed in Figure 5 .
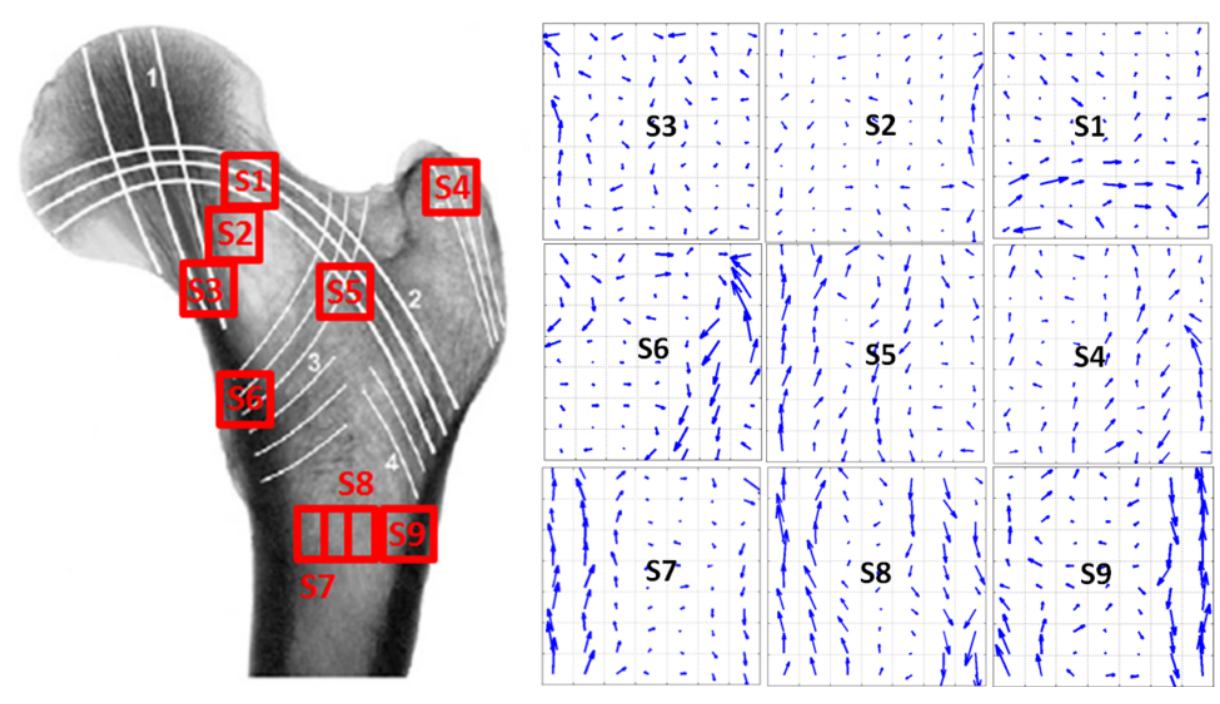

Fig. 4. Distribution of the first-principal elasticity modulus $\left(E_{1}=E_{\mathrm{a}}\right)$ over the sagittal middle-plane. 
Table 1

Differences (\%) between stresses/strains predicted by anisotropic and isotropic finite element models

\begin{tabular}{lll}
\hline & Maximum von Mises & Maximum First Principal Strain \\
\hline Femoral neck & 30.2 & 50.1 \\
\hline Intertrochanter & 12.3 & 49.8 \\
\hline Shaft & 8.1 & 0.8 \\
\hline
\end{tabular}
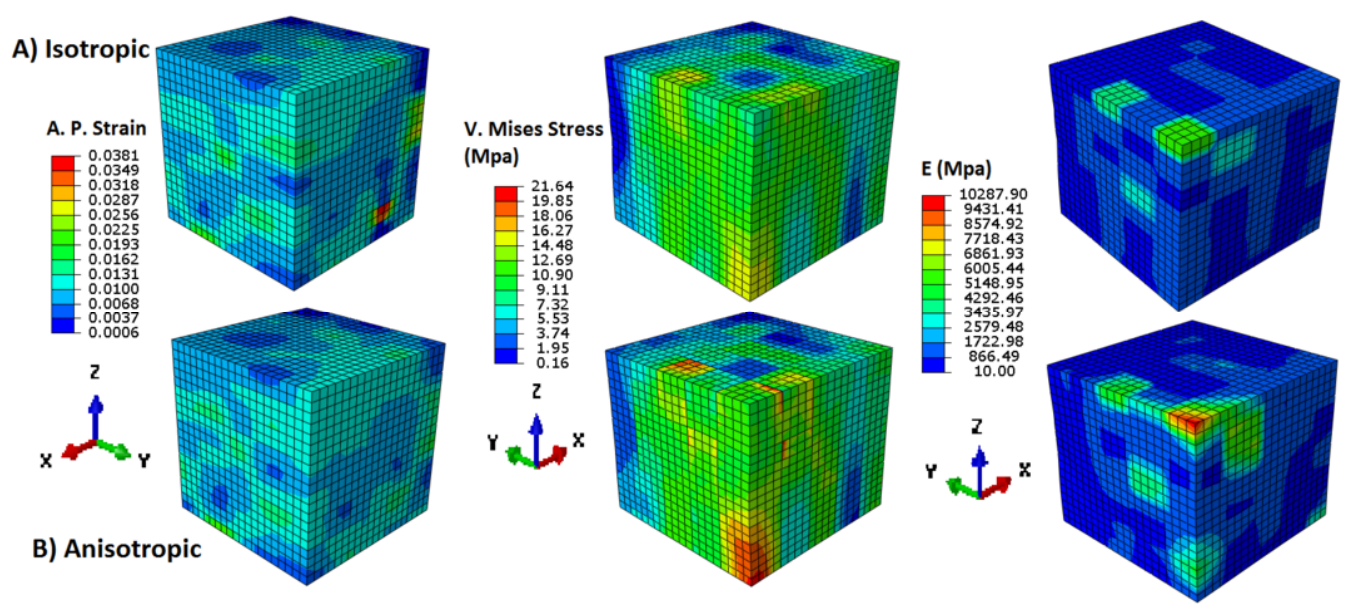

Fig. 5. Differences between A) anisotropic and B) isotropic finite element model with respect to the predicted first principal strain, von Mises stress, and elasticity modulus.

From the results presented in Table 1, it can be seen that the differences are location dependent. The largest differences occurred at the femoral neck, followed by those at the intertrochanter, and then those at the shaft. Trabecular bone is the dominant content in both of the femoral neck and the intertrochanter region, while the shaft is mainly composed of cortical bone. The results suggest that the adoption of an isotropic or anisotropic material model has more considerable effect on the trabecular bones than the cortical bones, with respect to the predicted stresses/strains. The above phenomenon can be briefly explained in the following. Under the action of compressive loading in the femur axial direction, the principal anisotropy orientation of the shaft cortical bone is roughly in the same direction of the loading, refer to Figure 4. The differences made by the material models was mainly from the magnitude of first principal elasticity modulus $\left(E_{1}\right)$. For the trabecular bones at the femoral neck and the intertrocnater, the first-principal anisotropy orientation has much larger deviation to the loading direction (Figure 4). Therefore, the differences in the trabecular were made by both of the magnitude and orientation of bone anisotropy. The obtained results may also indicate that trabecular bones have stronger anisotropy than cortical bones, but this phenomenon need be further investigated. Another aspect that is to be studied is the bone elasticity-density relation proposed in this paper. It need be tested by well-designed experiments.

\section{Conclusion}

In this preliminary study, the effect of adopting isotropic or anisotropic material model on the predicted stress/strain level in finite element models of bones is investigated. The anisotropic bone elas- 
ticity-density relation was obtained by assuming that bone anisotropy is generated by variations in bone density and by modifying existing empirical isotropic relations. The numerical investigation results indicated that, consideration of anisotropy in bone finite element models has considerable effects on the predicted stresses/strains, especially for trabecular bones.

\section{References}

[1] Y.H. An and R.A. Draughn, Mechanical Testing of Bone and the Bone-Implant Interface, CRC Press LLC, 2000.

[2] S.C. Cowin, The relationship between the elasticity tensor and the fabric tensor, Mechanics of Materials 4 (1985), 137147.

[3] J. Kabel et al., Constitutive relationships of fabric, density, and elastic properties in cancellous bone architecture, Bone 25 (1999), 481-486.

[4] R. Moreno, M. Borga and O. Smedby, Generalizing the mean intercept length tensor for gray-level images, Medical Physics 39 (2012), 4599-4612.

[5] A. Odgaard, E.B. Jensen and H.J.G. Gundersen, Estimation of structural anisotropy based on volume orientation: A new concept, Journal of Microscopy 157 (1990), 149-162.

[6] A. Odgaard et al., Fabric and elastic principal directions of cancellous bone are closely related, Journal of Biomechanics 30 (1997), 487-495.

[7] Z.A. Tabor and E. Rokita, Quantifying anisotropy of trabecular bone from gray-level images, Bone 40 (2007), 966-972.

[8] Z.A. Tabor, Estimating structural properties of trabecular bone from gray-level low-resolution images, Medical Engineering \& Physics 29 (2007), 110-119.

[9] D. Inglis and S. Pietruszczak, Characterization of anisotropy in porous media by means of linear intercept measurements, International Journal of Solids and Structures 40 (2003), 1243-1264.

[10] R.W. Goulet et al., The relationship between the structural and orthogonal compressive properties of trabecular bone, Journal of Biomechanics 27(1994), 375-389.

[11] J.Y. Rho, L. Kuhn-Spearing and P. Zioupos, Mechanical properties and the hierarchical structure of bone, Medical Engineering \& Physics 20 (1998), 92-102.

[12] J.Y. Rho, R.B. Ashman and C.H. Turner, Young's modulus of trabecular and cortical bone material: Ultrasonic and microtensile measurements, Journal of Biomechanics 26 (1993), 111-119.

[13] J.Y. Rho, M.C. Hobatho and R.B. Ashman, Relations of mechanical properties to density and CT numbers in human bone, Medical Engineering \& Physics 17 (1995), 347-355.

[14] K.J. Bundy, Experimental studies of the nonuniformity and anisotropy of human cortical bone, Ph.D. Dissertation, Stanford University, 1974.

[15] R. Schneider et al., Inhomogeneous, orthotropic material model for the cortical structure of long bones modelled on the basis of clinical CT or density data, Computer Methods in Applied Mechanics and Engineering 198 (2009), 2167-2174.

[16] J.C. Lotz, T.N. Gerhart and W.C. Hayes, Mechanical properties of metaphyseal bone in the proximal femur, Journal of Biomechanics 24 (1991), 317-329.

[17] J.C. Lotz, T.N. Gerhart and W.C. Hayes, Mechanical properties of trabecular bone from the proximal femur: A quantitative ct study, Journal of Computer Assisted Tomography 14 (1990), 107-114.

[18] Y. Luo, A local multivariate Lagrange interpolation method for constructing shape functions, International Journal for Numerical Methods in Biomedical Engineering 26 (2010), 252-261.

[19] B. Helgason et al., Mathematical relationships between bone density and mechanical properties: A literature review, Clinical Biomechanics 23 (2008), 135-146. 